\title{
EXTREMELY HIGH ENERGY COSMIC RAYS AND THE AUGER OBSERVATORY
}

\author{
MURAT BORATAV \\ LPNHE, Université Paris 6, 4 Place Jussieu \\ 75005 Paris, France \\ E-mail: boratav@in2p3.fr
}

\begin{abstract}
Over the last 30 years or so, a handful of events observed in ground-based cosmic ray detectors seem to have opened a new window in the field of high-energy astrophysics. These events have energies exceeding $5 \times 10^{19} \mathrm{eV}$ (the region of the so-called GreisenZatsepin-Kuzmin spectral cutoff) ; they seem to come from no known astrophysical source ; their chemical composition is mostly unknown ; no conventional accelerating mechanism is considered as being able to explain their production and propagation to earth. Only a dedicated detector can bring in the high-quality and statistically significant data needed to solve this long-lasting puzzle : this is the aim of the Auger Observatory project around which a world-wide collaboration is being mobilized.
\end{abstract}

\section{Introduction}

This article will mainly concern the problems raised by the existence and observation of cosmic rays whose energies are around and above $10^{20} \mathrm{eV}$ (or $100 \mathrm{EeV}$ ). Such cosmic rays -that we shall call the "extremely high energy cosmic rays" or EHECRare exceptional for the following reasons :

- Even when one takes into account the experimental uncertainties on their energy measurement (of the order of 20\%), they are well above the so-called GreisenZatsepin-Kuzmin (GZK) cutoff. This cutoff corresponds to the threshold for inelastic collisions between the cosmic microwave background (CMB) and protons (photo-pion production) or heavy nuclei (photo-disintegration). The result is that no EHECR observed on earth can be produced by sources very distant from us. We shall come later on this very important point.

- There are very few (if none) conventional astrophysical sources considered unanimously by the experts as being able to accelerate particles at energies exceeding those of the most energetic of the EHECRs that have been observed in the past.

- At such energies, the effect of the galactic and extra-galactic magnetic fields are quite weak on the EHECRs. Thus, even if those are charged particles, their reconstructed incident direction should point toward their sources within a few degrees. This distinguishes the EHECRs from their brothers in the lower energy regions : one can use them for point-like-source-search astronomy. 
In the following, we shall try to demonstrate that if one takes into account all the necessary conditions for an EHECR to be observed on earth (production energy, production rate, escape from production site, propagation, interaction with earth's atmosphere), the natural conclusion should be that the EHECRs do not exist. However, they exist and have been detected, although in small numbers : less than 50 events above $40 \mathrm{EeV}$, and only 9 with energies equal to or larger than $100 \mathrm{EeV}$. This is a very exciting situation that one could actually consider as being symmetrical with the present status of the dark matter problem : in one case (the dark matter) we deal with "particles which should exist and don't, while in the other (the EHECRs) with particles which do exist but perhaps shouldn't" [酉].

One last remark about the relevance of the Auger Observatory with the main topic of this conference : the neutrino telescopes. Other contributions [2] showed during this meeting that the detection of neutrino induced showers in the atmosphere could be a very interesting alternative to other techniques (telescopes immerged in water or ice) at the highest energies. On top of that, no decisive argument can rule out a neutrino component for the EHECRs ; actually, the neutrino hypothesis would make life easier when looking for a solution to the EHECR puzzle (no upper limit would be necessary for the distance of the source) although this would be at the cost of raising a new series of problems. We shall come back shortly on this aspect in the following.

\section{What do we know of the EHECRs?}

The first -and the most important- fact on these cosmic rays is that they exist! If we concentrate on those with energies equal to, or larger than, $10^{20} \mathrm{eV}$ (this is an arbitrary threshold, no physical reason justifies this special value), 9 events were observed in the past, with five different ground detectors: Volcano Ranch [3], Haverah Park [4], Yakutsk [5], Fly's Eye [6] and AGASA [17]. The detections were spread over more than 30 years and the detectors involved are all based on different detection techniques (scintillators with or without muon detection, air- and water-Cerenkov, atmospheric fluorescence). Thus, it is almost impossible to envisage that the detected events could result from experimental biases, artefacts or calibration errors.

The flux of these cosmic rays is extremely low at the energies we are interested in. This can be roughly parametrized by a simple law :

$$
I\left(E>E_{0}\right)=\frac{100}{E_{0}^{2}}
$$

in $\mathrm{km}^{-2}$.sterad ${ }^{-1} \cdot$ year $^{-1}$ when $E_{0}$ is given in EeV. The two most recent (and most energetic) of these events have energies of 200 and $320 \mathrm{EeV}$, with uncertainties of less than 30\% (including systematics).

Nothing decisive can be said on the chemical composition on these events. The only detector capable of providing a somewhat precise information on this feature is 
the Fly's Eye. Their data including the lower energy region of the spectrum is compatible with the interpretation that between 1 and $10 \mathrm{EeV}$ the chemical composition of the cosmic rays shifts gradually from heavy nuclei (e.g. Fe) to protons. A careful study of the highest energy event (the one at $320 \mathrm{EeV}$, also detected by the Fly's Eye) seems to exclude only a pure electromagnetic shower, the profile of the shower being compatible with that generated by a light or heavy hadron. A neutrino induced hadronic shower remains possible but this brings up the interaction probability of a neutrino with the higher atmospheric layers.

A first look at the reconstructed directions of the EHECRs seems to show no clear pattern indicating the existence neither of pointlike sources nor of large scale structures where such sources could be grouped. One should keep in mind that at such energies, and from what we know of the galactic and extragalactic magnetic fields, the EHECRs should point directly to their sources if these are reasonably close. The Larmor radius of a charged particle at $320 \mathrm{EeV}$ is larger than the size of the galaxy if its charge is less than 8 . If we take the currently accepted upper limit $\left(10^{-9} \mathrm{G}\right)$ for the extragalactic magnetic fields, a proton of the same energy should have a Larmor radius of $300 \mathrm{Mpc}$ or more, when the propagation arguments (see below) would exclude its source being at a distance larger than a few tens of Mpc.

A recent study [8] using the totality of the data available above $20 \mathrm{EeV}$ shows that there is a quite convincing correlation between the arrival directions of the subsample of events with energies above $40 \mathrm{EeV}$ and the supergalactic plane, a structure related to the local group and roughly perpendicular to the galactic plane. The authors note that this same plane is also the direction where there is an especially high density of radiogalaxies. It is premature to consider such an analysis as bringing any proof as for the nature of the accelerating mechanisms. Much larger statistics are needed for such statistical methods to be definitively conclusive.

Among the recent data published by the AGASA collaboration, there are three pairs of events whose directions are superimposed. If one assumes that these pairs come from common pointlike sources], interesting conclusions can be reached without appealing to any particular model [9]. For example, just by using the known values and pattern of the galactic magnetic fields, it can be shown that the detected cosmic rays cannot be anything except protons (if they are charged, of course). Then, setting the usual upper limits to the distance of the source, one can show that the limit of the average extragalactic magnetic field (with a hypothesis of $1 \mathrm{Mpc}$ cell size) is about $5 \times 10^{-10} \mathrm{G}$, a value very close to that obtained with the help of sophisticated analysis techniques such as Faraday rotation of polarized light coming from distant galaxies. Although one cannot say more than what is stated above about the common origin of overlapping events, this shows how rich the analysis of such events can be, provided there is a reasonable amount of them available.

\footnotetext{
${ }^{1}$ There is a $2 \%$ probability that the directions of the three pairs overlap just by chance.
} 


\section{Origin and transportation of the EHECRs}

We made previously the strong statement that the observed EHECRs should not exist. This needs no doubt some explication and proof. What we mean by this statement is the following. The observation of such a cosmic ray's interaction by a ground-based detector means that previously it went through a series of processes and trials which, all put together, seem almost impossible to satisfy the observed facts. One should use the adverb "almost" since we know that for a very small quantity of cases it did indeed happen. First the particle has to be accelerated at energies much larger than that measured when detected. For this we shall consider mainly what we call "conventional accelerating mechanisms" known in astrophysics. Then it has to escape from its production site : there one has to take into account "beamdump" effects which exist in the vicinity of most of the candidate sources and where a substantial part of the particle's energy is tapped off. Provided there is enough energy left, the particle has then to travel across the intergalactic medium where inelastic reactions with the cosmic microwave background (CMB) occur and degrade more of the particle's energy. And finally the cosmic ray interacts with the atmosphere creating an extensive air shower (EAS) whose physical characteristics have to be in conformity with what is observed in the few cases mentioned above. Any proposed scenario attempting to give an explanation on the origins of the EHECRs has to be checked against not only one (e.g. the first) of these steps but against the complete set of them : only then it can be taken seriously.

Whenever we shall need to illustrate our case by an explicit observation, we'll use the example of the champion of all observed cosmic rays, which is the one of $320 \mathrm{EeV}$ detected in 1992 by the Fly's Eye and whose study and interpretation generated an abundant litterature (see e.g. one of the most detailed of those articles [10]).

\subsection{The conventional accelerating mechanisms}

By conventional we mean accelerating mechanisms based on the electromagnetic fields existing inside or in the vicinity of known astrophysical objects. Whatever the acceleration mechanism, there is a necessary constraint which has to be verified by the "site" where this mechanism operates and which relates its size to the magnetic field existing there. This condition was pointed out years ago by M.Hillas [11] and can be understood in the following way. A particle which is progressively accelerated to the highest energies has to be contained inside an "acelerating site" during the whole process. This is only possible if its Larmor radius is smaller than the size of the site characterized by a radius $R$. This imposes a simple condition on the magnetic rigidity, which one can express as an easy-to-remember approximate formula :

$$
B R>\frac{E}{Z}
$$


where $Z$ is the charge number of the particle, $B$ is in $\mu \mathrm{G}, R$ in kpc and $E$ in EeV. Since this is a necessary condition, one can limit the search for candidate sources to those where the magnetic rigidity checks this relation. The popular and well known "Hillas plot" consists in positioning the known astrophysical objects in a figure where, in logarithmic scales, $R$ is plotted on the abscissa, $B$ in ordinates and diagonal straight lines show the wanted values of the energy as a function of $Z$. If one looks at sites where a proton can reach the energy of $100 \mathrm{EeV}$, one can see that very few possibilities remain above the corresponding line : powerful radio-galaxies in the large $R$-low $B$ region, neutron stars in the large $B$-low $R$ region and active galactic nuclei (AGN) in between. Although in all three cases the acceleration mechanism is different, these are the most powerful candidate accelerators in the universe. However, there is no general agreement in the community that any one of these accelerators is able to reach energies in the $\mathrm{ZeV}$ range $\left(\mathrm{Z}\right.$ is for zetta $=10^{21} \mathrm{eV}$ ), energies necessary if one wants to understand the existence of cosmic rays detected at $320 \mathrm{EeV}$. Most predictions usually limit the accelerating power of such systems to a maximum of 10 to $100 \mathrm{EeV}$. However, the most optimistic authors do not exclude the possibility that a proton may be accelerated at energies of $1 \mathrm{ZeV}$ by diffusive shock acceleration in quasars and radiogalaxies [13].

Now, suppose that this is possible. Then at least for two of the sites appears, among others, the problem of the "beam-dump" encountered by the particle as soon as it is accelerated. For the neutron star, this is precisely the accelerating (moving) magnetic fields themselves : the particle is expected to lose most of its energy by synchrotron radiation when crossing these fields on its escape trajectory. For the AGN, the dump consists in the very intense radiation fields surrounding the central parts of those engines. At very high energies, the energy loss in such regions are the result of processes similar to those we shall describe in the next paragraph.

\subsection{From the source to the earth}

Suppose again that by some kind of miracle, the preceding obstacles are overcome, and the particle starts its long journey to earth. It has to go through a medium filled (with a density of about 400 photons $/ \mathrm{cm}^{3}$ ) with the CMB, photons with an average energy of about $10^{-3} \mathrm{eV}$. If we blue-shift the photon to the, say, proton's rest system, we see that we are dealing with inelastic photoproduction processes (photopion production) provided the proton has an energy in excess of about $5 \times 10^{19} \mathrm{eV}$. The back-of-the-envelope formula giving the energy of the $3 \mathrm{~K}$ photon in the rest frame of a proton of $100 \mathrm{EeV}$ is :

$$
E(\mathrm{MeV}) \approx \frac{25}{\lambda(\mathrm{cm})}
$$

which gives a few hundreds of $\mathrm{MeV}$ since the $3 \mathrm{~K}$ photons have millimetric wavelengths. Such cross-sections have quite large values (fraction of millibarn). The mean free path 
of a $300 \mathrm{EeV}$ proton is about $6 \mathrm{Mpc}$ [12 and each interaction with the $3 \mathrm{~K}$ photons degrades the proton's energy by more than $10 \%$. This means that the proton very quickly loses its energy until this goes below the inelastic threshold. The consequence is that whatever the energy with which the proton started, after at most $100 \mathrm{Mpc}$ or so its energy is found to be less than $100 \mathrm{EeV}$. In other terms, if the $300 \mathrm{EeV}$ cosmic ray is a proton, its source cannot be further than a few tens of Mpc. This is the origin of the spectral GZK cutoff [14].

One could object that there is no reason that the EHECRs have to be protons, which is right. But one can see that the same kind of conclusions are reached with any kind of particle, except neutrinos. If one considers the possibility of these cosmic rays to be photons (which hypothesis should not be excluded a priori) the interactions with the background radiations (including the radio waves) still limit the range very drastically. The pair creation threshold is around $300 \mathrm{TeV}$ for the incident photon energy and the mean-free-path of a $320 \mathrm{EeV}$ photon would be $20 \mathrm{Mpc}$ with an asymptotic limit of about $30 \mathrm{Mpc}$ [12]. Another way of seeing the problem is a thumb-rule giving the survival probability for a photon of $300 \mathrm{EeV}$ after a trajectory of $L \mathrm{Mpc}$ :

$$
P(L) \approx \mathrm{e}^{-\mathrm{L} / 6.6}
$$

which yields a probability of less than $10^{-3}$ for a distance larger than $50 \mathrm{Mpc}$.

Can the EHECRs be heavy nuclei? We already said that the observed events at $E>100 \mathrm{EeV}$ do not favour this hypothesis but cannot exclude it either. However, here again, the propagation processes put very stringent conditions on the distance to the source. The photodisintegration reactions with the CMB are expected to strip on average four nucleons per Mpc from the traveling nucleus. If a heavy nucleus (iron, oxygen) is produced with an initial energy of, say $800 \mathrm{EeV}$, after less than $10 \mathrm{Mpc}$ the energy of the surviving fragment goes below $300 \mathrm{EeV}$ and reaches the $100 \mathrm{EeV}$ threshold at around $20 \mathrm{Mpc}$ [14. The clear conlusion is the following : if the highest energy cosmic rays observed were heavy nuclei, they could only be of galactic origin. In this case, even with large charges (hence small Larmor radii) some anisotropy in their direction should be easily detectable with a resonable amount of statistics.

The overall conclusion from the arguments above is that the potential source for the highest energy cosmic rays observed cannot be situated at distances much larger than a few tens of Mpc (i.e. probably within the local cluster of galaxies), except if these cosmic rays are neutrinos (immune to interactions with the CMB). Since at such energies, the reconstructed directions should point clearly toward the source (except for heavy nuclei), it is easy to look for astrophysical objects in the categories mentioned in the previous paragraph contained in a sphere of, say, $50 \mathrm{Mpc}$ around us. This exercice was made for the directions of the highest energy cosmic rays (especially for the $320 \mathrm{EeV}$ Fly's Eye event) and no candidate source was found.

Can the EHECRs be neutrinos? This is a very interesting hypothesis since it kills all arguments limiting the distance of the source. Several authors studied this 
possibility 113, 10, 12. The answer is "yes" but with many question marks. First, the neutrino induced shower has to reproduce the features of the observed EAS. For this, the Fly's Eye detector can be powerfully discriminating since it observes the longitudinal development of the shower and can trace back the shower's origin. These have very caracteristic features depending on the initial particle. If the incident particles were neutrinos, not only would we need very large fluxes to explain the few observed events but also we would expect those to interact with a flat distribution in atmospheric depth. However one can answer this objection by another : "Who knows how a 100 EeV neutrino actually interacts with nuclei?" If one takes seriously such an argument, it would mean that the EHECRs open a new window in particle physics. Unfortunately in this case, we can see no obvious method for distinguishing neutrino showers from their hadronic or electromagnetic partners. If one stays within the limits of the standard model, there is an easy way for a ground detector to discriminate neutrino showers from the rest. We'll say a few words about the ability of the Auger Observatory to detect EHE neutrinos in the last paragraph.

Supposing we ignore the distance constraint for the $320 \mathrm{EeV}$ event (maybe invoking the neutrino hypothesis). One has still to answer some questions of astrophysical origin. As an example, it has been noted [10] that there is a very powerful Seyfert galaxy (MCG 8-11-11) in the approximate direction of this event but at a distance of $900 \mathrm{Mpc}$. It is however impossible to explain why such a source capable of producing a $320 \mathrm{EeV}$ particle would give no clear signal (easily visible by the Fly's Eye detector) in the $10 \mathrm{EeV}$ region where the attenuation with the distance even for protons is much weaker.

Thus the overall comment on what precedes is what was announced in the beginning : when all the necessary processes and observational facts have been taken into account, no globally acceptable explanation is found for the existence and detection of the EHECRs.

\subsection{The exotic sources}

Before beginning the description of the dedicated experimental project, it is worthwhile to say a few words about some non-conventional ideas on the possible sources of EHECRs.

In their search for models explaining large scale structure formation in the universe, cosmologists have produced a class of appealing models where appear objects called "topological defects" 15. The cosmic strings are considered the most likely of the topological defects to be observed some day. The production of these defects are initially linked to phase transitions in the early universe but present day mechanisms producing such objects seem not to be excluded. A cosmic string is a region of space where huge energy densities $\left(10^{22} \mathrm{~g} / \mathrm{cm}\right)$ are present. Such regions could trap grand-unification particles of mass up to $10^{24} \mathrm{eV}$. In some cases (two crossing strings, 
collapse of a string on itself), the GU particles would be liberated. Since they are unstable, they would immediately disintegrate and emit large numbers of ordinary particles whose energies could easily exceed those of the EHECRs. Some authors even envisage that the cosmic string itself could interact with the atmosphere and initiate the EAS.

This very speculative hypothesis is also very attractive because it is practically the only one which would survive if it is confirmed that the sources of the EHECRs are indeed very close and diffuse.

Many authors [13, 16, 17] checked the cosmic string hypothesis against the experimental data. Although some hypotheses in this sector have to be abandoned, such as gauge strings as being responsible of the observed EHECRs [16], many models remain viable and testable (e.g. by high rates of photon component in the EHECRs) and may well open a new window in cosmology and particle physics.

\section{The Auger Observatory}

The present day situation as regards the highest energy part of the cosmic ray spectrum, the many unknowns as to the origin of these cosmic rays, the posibility that those events may open new windows in cosmology, astrophysics or particle physics, all these are strong arguments in favour of a dedicated experiment. What can one ask of such a project? The first and most important requirement is that it accumulates statistics. It is hopeless to expect any element of answer to this astrophysical puzzle if the detection rate of the relevant events goes on at the present level, which means several more decades to have a few tens of events above the GZK cutoff. Then, the projected detector must be able to measure the energy of the incoming cosmic rays with a better resolution (say, 10\%) than what is available now. It must have a good resolution in measuring the direction of the cosmic ray since, as was said above, we'll be doing astronomy with these particles. And finally, the identity of the incident particles (or the chemical composition if those are a mixture of several species) must be detected on a statistical, if not individual, basis.

The Auger observatoryf is precisely designed to fulfill the whole set of those requirements. We'll give a brief description of this detector and of its expected performance, and advise the interested reader to consult a detailed technical report written by a working group of many people during the first six months of 1995 18.

\footnotetext{
${ }^{2}$ Pierre Auger, a french physicist who played an important role in the founding of CERN, was the first to have the intuition that cosmic ray events observed coincidentally in distant detectors could be extensive air showers of very high energy.
} 


\subsection{Description of the detector}

The Auger observatory is a "hybrid" ground detector which will be installed on two sites, respectively in the southern and northern hemisphere. The aim of having two components to the detector is to be able to see the whole sky. The southern hemisphere detector is especially interesting since very few detectors took data in the past in this part of the world from where the direction of the center of our galaxy is visible.

The detector will be designed to be fully efficient for showers with energies of 10 $\mathrm{EeV}$ and above. This will make the link with the part of the energy spectrum well explored by presently operating detectors, especially AGASA and Fly's Eye.

Each site will be equipped with an array of "stations" covering an area of about $3000 \mathrm{~km}^{2}$. Thus, the total surface of $6000 \mathrm{~km}^{2}$ is expected to yield a rate of several thousands of events detected per year above $10 \mathrm{EeV}$, and between 50 and 100 above $100 \mathrm{EeV}$. Each giant array will be completed by an optical device detecting the fluorescence light emitted by the nitrogen molecules of the atmosphere excited by the charged particles produced in the EAS. The northern site should host a detector derived from the japanese Telescope Array project [19]. The southern site will be equipped with an improved version of the Utah University's Fly's Eye [18, 20]. The reason for the detector to be "hybrid" is that, for about $10 \%$ of the events jointly detected by the array and the optical device, not only the quality of the data will be much improved but also each detector will be used to cross-calibrate the other since they measure independently and with different methods all the shower's parameters.

The individual stations will be water-Cerenkov tanks where the Cerenkov radiation emitted by the charged particles penetrating in the detector will be read out by three phototubes. The output signal will be digitized by rapid cycling flash ADCs, whose aim is to separate the electromagnetic signal (low energy photons and electrons) from the muons crossing the tank. The relative synchronization of the stations (with a few ns precision) will be done using signals emitted by GPS (Global Positioning System) satellites. The communication between stations and the data transfer to a central computer will be done by using radio signals by methods similar to cellular telephone techniques. The stations will be powered with solar panels and batteries.

The constraints on the site come from the above specifications. The lattitude should be around $40^{\circ}$ North and South. The array needs a $3000 \mathrm{~km}^{2}$ flat region (lineof-sight visibility between neighbouring stations for radio reception) with moderate temperatures (water tanks) and cloudless skies (solar panels). The optical detector needs a transparent atmosphere and no source of light pollution nearby.

Among half a dozen sites visited by a survey team in the southern hemisphere, the region of las Leñas (north of Patagonia in Argentina) was chosen by the collaboration during a full meeting in November 1995. The northern site will be chosen in September 1996. 


\subsection{The direction of the primary}

In the optical detectors, pixels seeing just a small portion of space record the time of arrival of light pulses in this direction. The array registers the time of arrival of the particles in the water tanks. This timing information enables both detectors (with different methods) to reconstruct the direction of the shower axis, hence the direction of the incident particle. For showers close to the vertical, the resolution for the array alone is of the order of $2^{\circ}$ or better above $10 \mathrm{EeV}$. For hybrid detection of showers, this resolution can reach $0.2^{\circ}$.

\subsection{The energy measurement}

On average, a charged particles deposits, for every depth equivalent of $1 \mathrm{~g} / \mathrm{cm}^{2}$, an energy of $2.2 \mathrm{MeV}$. The integral of this energy deposit converted into fluorescent light is seen by the optical detector as the longitudinal development of the shower. A particularly important parameter measured by this detector is the position of the shower maximum $X_{\max }$. The total detected light and $X_{\max }$ are the two parameters through which the total energy of the shower can be estimated. In the 10-100 EeV range, the Fly's Eye energy resolution is of the order of $30 \%$ including statistical fluctuations and systematic uncertainties.

The ground array usually measures the energy of the shower from the lateral density distribution of particles. There is a "magic" parameter coming from lower energy methodology, the density at $600 \mathrm{~m}$ from the shower core, which was shown to be the best estimator, through a rough proportionality law, of the incident particle energy. Actually, this distance is simply the one where fluctuations due to the shower tail and those coming from the lateral distribution partly compensate and the total fluctuation becomes minimum. The density at this distance is quite weakly dependent on the nature of the initial particle. At higher energies relevant to Auger, this distance seems to be slightly larger. The estimation of the total energy in Auger should be improved by the fact that the detector will be able to measure separately the muon and electromagnetic densities. For hybrid measurements, we expect the energy resolution to be better than $10 \%$ at $100 \mathrm{EeV}$.

\subsection{Identification of the primary}

The main feature which differentiates showers coming from heavy or light nuclei is the depth at which occurs the shower maximum $X_{\max }$. Shower development studies showed that a shower from a heavy nucleus behaves more or less as a superposition of showers coming from the individual nucleons of the nucleus sharing the total energy between them. This explains why the shower maximum is at a higher altitude for heavy nuclei and also why those have a larger muon component at the ground level (the pions reach faster the low energy limit below which they decay before interacting). 
Heavier nuclei produce also faster risetimes (the rate at which the particles reach the ground at a given distance from the shower core). Those are the three parameters one can use to identify the primaries : the time structure of the ground particles, muon to electromagnetic densities as a function of the distance to the core, the position of the shower maximum. The first two can be measured by the ground array, the last by the optical device, hence providing two independent methods to identify the primary. The simulations show that if the muon to photon/electron separation in the Cerenkov detector is good enough, the primary composition should be measured on a statistical basis if not for individual showers.

\subsection{The detection of neutrinos}

Since this is the main topic of this conference, let us show that, at least for the highest energies, Auger should perform as a competitive neutrino detector. The neutrino astronomy above $10 \mathrm{EeV}$ is directly related to the GZK spectral cutoff : this is the only remaining hypothesis if one excludes the possibility that the EHECR sources are actually situated within a few tens of Mpc.

The Fly's Eye group [17] have studied the possibility for this detector (the HiRes version) to observe neutrinos from topological defects. The results depend strongly of course on the incident fluxes, hence of models, but a few events of this type are expected for a long term experiment. The aperture of the HiRes detector for neutrinos with energies of the order of $100 \mathrm{EeV}$ is expected to be between $10^{4}$ and $10^{5} \mathrm{~m}^{2}$.sr. Of course, the detection rate suffers from the irreducible $10 \%$ duty cycle of any optical detector whose operation is limited to dark moonless nights and clear skies.

Cronin, Parente and Zas have recently studied the possibility for the ground array of detecting horizontal showers. Their partial results were presented at this workshop by E.Zas. Let us just remind that the water Cerenkov tanks are sensitive to charged particles, whatever their direction. Thus for horizontal air showers (HAS), provided their axis is at a reasonable altitude (say, less than $3 \mathrm{~km}$ ) above the ground level, the Auger array behaves as a calorimeter with uniform density of matter and longitudinal sampling. Showers with directions at very large angles, if observed, can hardly be anything except neutrino induced (the horizontal atmosphere thickness is roughly 36 times the vertical one). The component due to hard muon bremsstrahlung can easily be eliminated with an appropriate trigger or off-line analysis. If the neutrino interaction (which should be uniformly distributed) happens to be close enough to the array so that the shower maximum falls within the instrumented area, then the array becomes a very powerful detector since it samples the totality of the shower components, provided the neutrino rates are appropriate. Those were studied by the above mentioned authors and the possibility that the Auger array may detect a few tens of neutrino induced HAS above $10 \mathrm{EeV}$ per year seems realistic. In any case, independent of the incident fluxes, one figure to keep in mind is that the Auger array 
has an acceptance of $10 \mathrm{~km}^{3}$.sr water equivalent for HAS of $10 \mathrm{EeV}$, this acceptance being a slowly increasing function of the energy.

\section{Conclusion}

The projected Auger observatory is presently in the R\&D phase where all the technical issues developed in the design report 18 are being optimized and tested. About 150 physicists and engineers from 15 countries are working on this project just now. Its scientific aims are very clearly established : this is an observatory (very much as an astronomical one) optimized for the detection of cosmic rays with energies above $10^{19} \mathrm{eV}$. Such events, if detected in large quantities, may enable us to answer one of the very rare (hence very exciting) open questions in the field of astrophysics and/or cosmology : what are the sources of the most energetic particles observed in the universe, i.e. the most powerful machines existing in Nature?

Skimming through the steady flow of recent articles related to high energy astrophysics, one can easily see that this physics issue may have many implications, i.e. it may be related to a variety of fields where the (particle) astrophysics community is quite active : AGNs and pulsars as powerful accelerating engines, cosmic strings or other topological defects, gamma ray bursts [21], neutrino astronomy etc...

Finally, one should be aware that this project is also open to people not primarily interested in its specific physics motivation, but who could consider it as an available experimental "facility". The Auger observatory provides two fully equipped areas of $3000 \mathrm{~km}^{2}$ each with possibility of very precise synchronisation over the whole surface, telecommunication equipments to communicate and exchange data, a central station equipped with a computer center and connections to high-flow international networks and real-time access to data from any part of the world. The capacity of such a detector to work with other types of equipments is limited only by the imagination of the potential users.

\section{References}

[1] L. M. Celnikier, Proc. of the Rencontres de Moriond, February 1996 (to be published).

[2] F. Halzen and E. Zas, these proceedings.

[3] J. Linsley, Phys. Rev. Lett. 10 (1963) 146.

[4] M. A. Lawrence, R. J. O. Reid and A. A. Watson, J. Phys. G. 17 (1991) 773.

[5] B. N. Afanasiev et al, Proc. of the 24th ICRC, Rome, Italy 2 (1995) 756. 
[6] D. J. Bird et al, Ap. J. 424 (1994) 491.

[7] S. Yoshida et al, Astropart. Phys. 3 (1995).

[8] T. Stanev et al, Phys. Rev. Lett. 75 (1995) 3056.

[9] J. W. Cronin, private communication.

[10] J. W. Elbert \& P. Sommers, Ap. J. 441 (1995) 151.

[11] A. M. Hillas, Annual Review Astron. Astrophys. 22 (1984) 425.

[12] F. Halzen et al, Astropart. Phys. 2 (1995) 151.

[13] G. Sigl, D. N. Schramm \& P. Bhattacharjee, Astrop. Phys. 2 (1994) 401.

[14] For a detailed discussion on the transportation of high-energy protons through the CMB, see e.g. F. A. Aharonian \& J. W. Cronin, Phys. Rev. D50 (1994) 1892.

[15] For a recent, complete and comprehensive review on the subject, see e.g. A. Vilenkin \& E. P. S. Shellard, Cosmic strings and other topological defects (Cambridge University Press, 1994).

[16] A. J. Gill \& T. W. B. Kibble, Phys. Rev. D50 (1994) 3660.

[17] S. Yoshida et al, Proceedings of the 24th Int. Cosmic Ray Conf., Rome (1995) vol. 1, p. 793.

[18] The Pierre Auger Project Design Report, Fermilab, October 1995.

[19] M. Teshima et al, Tokyo Workshop on Techniques for the Study of Extremely High Energy Cosmic Rays, ICRR - Tokyo (September 1993), p. 109.

[20] P. Sokolsky, P. Sommers \& B. R. Dawson, Phys. Rep. 217 No.5 (1992) 225.

[21] E. Waxman \& P. Coppi, Ap. J. Lett. (in press)

J. Miralda-Escudé \& E. Waxman, submitted to the Ap. J. Lett. 